\title{
OBSERVATIONS ON THE EFFECT OF ANOXEMIA ON THE HEART
}

III. Changes in the Auricles, with Particular Reference to the Relationship Between ANoxemia and Auricular Fibrillation

BY WILLIAM H. RESNIK

(From The Cardiographic Laboratory of the Medical Clinic of the Johns Hopkins Hospital and University)

(Received for publication August 6, 1925)

INTRODUCTION

In an earlier paper (Resnik, 1924), I reported several cases in which transient auricular fibrillation occurred as a result of the administration of digitalis. From observation of the response of these patients to atropine, the conclusion was reached that in some instances the drug acted directly on the myocardium. This was apparently at variance with experimental data of Lewis, Drury, Wedd and Iliescu (1921), who showed that strophanthin, acting directly on the auricle of the dog, hindered the development of auricular fibrillation, by increasing the absolute refractory period of the auricle. I suggested, therefore, that possibly the functional changes associated with myocardial failure, in which condition anoxemia is practically constant, may have so altered the auricular muscle as to permit the onset of auricular fibrillation, in spite of the increase in the absolute refractory period by digitalis. Other clinical and experimental observations have been reported which suggest that anoxemia predisposes the auricles to fibrillation. Vaquez (1911) mentions the appearance of transient auricular fibrillation in patients with myocardial failure, the arrythmia disappearing with circulatory improvement. Transient auricular fibrillation is relatively common in lobar pneumonia, being present in about 3 to 5 per cent of patients with this disease (Cole, 1920), one in which anoxemia is often a prominent symptom, but in which significant heart lesions are not usual (Cohn and Jamieson, 
1920). Although it is true that in lobar pneumonia the influence of a toxin is to be considered, I am unable to find that in other types of pneumococcus infections in which the same toxin should be at work, auricular fibrillation develops in the same percentage of cases as is true in lobar pneumonia. Experimentally, de Boer (1921) has shown that in the bled frog's heart, in which, among other nutritional disturbances, lack of oxygen must be assumed to be present, properly timed stimuli may cause the onset of fibrillation, whereas in the normally nourished heart, this arrythmia does not appear.

With these data in mind, suggestive but not conclusive, since in each of these examples there are other factors besides anoxemia which may play a role, I have attempted to determine more definitely the relationship between anoxemia and auricular fibrillation. I have already pointed out (Resnik, 1925) that ventricular fibrillation is prone to develop when the heart has been exposed to anoxemia.

\section{EXPERIMENTAL METHODS}

These have been described in a former communication (Resnik. 1925a) and it is only necessary to describe those procedures which have not been mentioned before. In order to determine the relative susceptibility of the auricles to develop fibrillation in periods of normal and low oxygen saturation of the arterial blood, ${ }^{1}$ the effect of faradic stimulation of the auricles was observed. In all experiments the vagus nerves were tied, and in all but one (experiment 35 ) the heart was fully under the influence of atropine. Throughout an experiment, the strength of the stimulating current, always well above the threshhold value, was unchanged, as was the duration of each stimulation, usually 15 to 30 seconds.

The method and precautions used in determining the refractory period of the atropinized auricles were those described by Lewis, Drury and Bulger (1921); break shocks were used exclusively as rhythmic stimulating and testing shocks. The timing device of Dieuaide and Turner (1924) was used to facilitate the refractory period determina-

${ }^{1}$ In all further mention of the oxygen saturation of the blood, either in the text or in the tables and figures, it will be understood that it is to the arterial blood that the reference is made. 
tions. In a few of the earlier experiments, two galvanometers were used simultaneously, one to record the rhythmic and interrupting stimuli, the other to record the responses of the auricular muscle. Later, only one galvanometer was available. It was found that with a sufficiently strong current, the signals were plainly visible on the film recording auricular activity, so that satisfactory measurements were possible by using one galvanometer alone, those records only from which accurate determinations could be made being used.

\section{RESULTS}

Effect of anoxemia on rate of impulse formation at the sino-auricular node. The results which I obtained were in agreement with those that have been observed by others, for there was first an acceleration

TABLE 1

Dog 34. Vagus nerves tied; fully atropinized

\begin{tabular}{c|c|c}
\hline Time & Oxygen saturation & Natural rate of heart \\
\cline { 2 - 3 } $11: 01$ & per cent & per minute \\
$11: 26$ & 100 & 205 \\
$11: 32$ & 90 & \\
$11: 46$ & 90 & 212 \\
$11: 53$ & 90 & 206 \\
$12: 03$ & 90 & 197 \\
$12: 03$ & 90 & 185 \\
\hline
\end{tabular}

and then a depression of the natural rate of the heart. Table 1 is illustrative of the response of the sino-auricular node to anoxemia.

Following a slight acceleration, the rate of the heart decreased gradually as anoxemia persisted until the condition of the animal was impaired, when the rate slowed rapidly. On one occasion so-called sino-auricular block was produced. The period of acceleration was usually of brief duration, and the stage of depression was generally seen at the time when auriculo-ventricular conduction was quickened. This difference in the behavior of the sino-auricular and auriculoventricular tissues is shown in table 2.

When anoxemia was relatively mild, re-establishment of the normal oxygen saturation of the blood was associated with a return of the heart rate to the normal level. When anoxemia was more severe 
and prolonged, however, the rate tended to remain low even when other functions, such as auriculo-ventricular conduction, became normal. In other words, the sino-auricular node is apparently highly sensitive to anoxemia: the stage of depression appears rapidly and recovery is slow.

I have discussed elsewhere (Resnik, (1925a)), the question of stimulation of sympathetic nerves in anoxemia. Although the acceleration in cardiac rate may, in part, be due to this, it seems clear that stimulation of sympathetic nerves does not entirely account for the increased heart rate. In one animal (experiment 31), in addition to tying both vagi and administering full doses of atropine, both stellate ganglia were removed. The heart rate was 163 per minute when the arterial

TABLE 2

Dog 15. Vagus nerves tied; fully atropinized

\begin{tabular}{c|c|c|c}
\hline Time & Oxygen saturation & Natural rate of heart & P-R interval \\
\cline { 2 - 3 } & per cent & per minute & second \\
$11: 33$ & 100 & 162 & 0.080 \\
$11: 47$ & 81 & & \\
$11: 57$ & 81 & 154 & 0.075 \\
$12: 30$ & 81 & 158 & 0.073 \\
$1: 15$ & 81 & 156 & 0.072 \\
$2: 01$ & 81 & 144 & 0.067 \\
$2: 16$ & 97 & & \\
$3: 01$ & 97 & 162 & 0.070 \\
\hline
\end{tabular}

blood was completely saturated with oxygen; fourteen minutes after a moderate anoxemia (oxygen saturation 85 per cent) was established, the rate rose to 172 per minute. The increase must have been due to a direct effect of anoxemia on the myocardium.

Faradic stimulation of the auricles during anoxemia. It is well known that under ordinary conditions faradic stimulation of the auricles leads almost constantly to the development of auricular fibrillation. If atropine is previously administered, however, in doses sufficient to paralyze the vagal endings, it is difficult, and sometimes impossible, owing to the prolongation of the absolute refractory period of the auricles by this drug, to produce auricular fibrillation by faradic stimulation (Winterberg, 1908). In eleven experiments I have compared the effect produced during periods of normal and low 
saturations of oxygen, of faradic stimulation of the auricles. In ten of these observations, vagal tone was completely removed, by tying both vagus nerves and giving frequent large doses of atropine. In one further experiment (experiment 35) atropine was not given, and under these conditions it may be assumed that vagal influence was largely but not entirely removed, since the vagal nerve endings were capable of being stimulated by the faradic current.

A strong faradic current was thrown into the auricles, the stimulating electrodes being placed at or near the junction of the superior vena cava with the upper end of the body of the auricle, and the duration of the after-effect was noted. This procedure was carried out a number of times, so that a clear idea of the susceptibility of the auricles to fibrillate could be obtained. If fibrillation ${ }^{2}$ resulted and lasted for more than one and a half to two minutes, or sometimes longer, faradic stimulation was employed to bring the circus movement to an end. This was done in order that more numerous observations could be obtained.

Using this method, I found that in six of the eleven experiments, there was a distinctly greater tendency for fibrillation to appear after the cessation of faradic stimulation during anoxemia than during normal oxygen saturation of the blood. In one experiment, ${ }^{3}$ evidence to the same effect was suggestive but not conclusive. In three further experiments there was no preponderating tendency in either anoxemia or normal oxygen saturation of the blood for auricular fibrillation to develop. Finally, in one experiment, there was a greater tendency for fibrillation to develop in the period of normal oxygen saturation of the blood than during anoxemia.

In table 3 are given the data of an experiment which illustrates

${ }^{2}$ In this connection, I shall mean "fibrillation" to express both fibrillation and flutter, since the work of Lewis and his associates has demonstrated that both these types of arrythmia have a common underlying mechanism.

${ }^{3}$ Two other experiments may be classed as being suggestive of the influence of anoxemia in predisposing the auricles to fibrillation. Thus, in one, no after-effect was observed after 25 stimulations with a faradic current. When anoxemia was instituted, fibrillation appeared after the third faradic stimulation and lasted for five minutes. No further attempts were made to note the effect of faradic stimulation, as other information was being sought. 
the type in which anoxemia favored the development of auricular fibrillation.

In this experiment, ten trials with the faradic current failed to produce auricular fibrillation when the blood was normally saturated with oxygen. Severe anoxemia was started; shortly afterwards, following three out of four faradic stimulations, fibrillation developed. Each period of fibrillation lasted about three minutes and ended when further faradic currents were thrown into the auricles. On restoration of normal oxygen saturation of the blood, three further trials failed to produce auricular fibrillation.

In table 4 is summarized another experiment of the same kind. It is given in greater detail, since it exhibits certain features which are worthy of mention.

TABLE 3

Dog 8

\begin{tabular}{c|c|c}
\hline Oxygen saturation & $\begin{array}{c}\text { Number of faradic } \\
\text { stimulations }\end{array}$ & Number of after-effects \\
\hline per cent & 10 & 0 \\
90 & 4 & $3^{*}$ \\
33 & 3 & 0 \\
91 & 10 & \\
\hline
\end{tabular}

* Each of these lasted about three minutes and was ended by faradic stimulation.

In this experiment, as in the one previously described, there was a definitely greater tendency for after-effects to appear during anoxemia than during the first period of normal oxygen saturation of the blood. When a high oxygen mixture was given following the preceding anoxemia, the auricles continued to exhibit for a short time a predisposition to fibrillation, which, however, disappeared later. That is to say, immediately after the cessation of anoxemia, the auricles behaved as if they had not fully recovered, and this I have observed on several other occasions.

Experiment 35 (table 5) illustrates another point of interest. The vagus nerves of the animal were tied; atropine was not used. The after-effects are classified in the table as short ( 5 to 59 seconds) and long (more than one minute). Fifty trials with faradic stimulation, five times associated with vagal stimulation, were ineffective in causing fibrillation during the first period of normal arterial oxygen 
TABLE 4

Dog 10

\begin{tabular}{|c|c|c|}
\hline Oxygen saturation & Faradic stimulation & Duration of after-effect \\
\hline per cent & & second \\
\hline \multirow[t]{10}{*}{94.5} & 1 & $0^{*}$ \\
\hline & 2 & 0 \\
\hline & 3 & 0 \\
\hline & 4 & 0 \\
\hline & 5 & 1 minute \\
\hline & 6 & 0 \\
\hline & 7 & $\mathbf{0}$ \\
\hline & 8 & 0 \\
\hline & 9 & 0 \\
\hline & 10 & 0 \\
\hline \multirow[t]{11}{*}{85} & 1 & 30 \\
\hline & 2 & $\mathbf{0}$ \\
\hline & 3 & 20 \\
\hline & 4 & 15 \\
\hline & 5 & 15 \\
\hline & 6 & 3 minutes $\dagger$ \\
\hline & 7 & 0 \\
\hline & 8 & 10 \\
\hline & 9 & 0 \\
\hline & 10 & 2 minute; \\
\hline & 11 & 0 \\
\hline \multirow[t]{12}{*}{96} & 1 & 1 minute \\
\hline & 2 & 0 \\
\hline & 3 & 0 \\
\hline & 4 & 3 minutes $\dagger$ \\
\hline & 5 & $\mathbf{0}$ \\
\hline & 6 & $\mathbf{0}$ \\
\hline & 7 & 10 \\
\hline & 8 & $\mathbf{0}$ \\
\hline & 9 & 0 \\
\hline & 10 & 0 \\
\hline & 11 & $\mathbf{0}$ \\
\hline & 12 & 0 \\
\hline
\end{tabular}

* After-effects of less than five seconds duration are recorded as 0.

$\dagger$ Ended by faradic stimulation.

saturation. A moderately severe anoxemia was produced, and in eight attempts, all without vagal stimulation, there resulted one 
short and one long after-effect. On return to a high oxygen mixture, five attempts, three with vagal stimulation, failed to bring on fibrillation. Anoxemia was again started, and this time five trials, associated with vagal stimulation, caused an after-effect to appear in each instance; the last one, of 44 minutes duration, ceased when normal oxygen saturation of the blood was restored. At this time, with a high oxygen mixture, two of four faradic stimulations, each combined with vagal stimulation, produced auricular fibrillation. Finally, when a severe anoxemia was started, seven trials failed to cause an aftereffect.

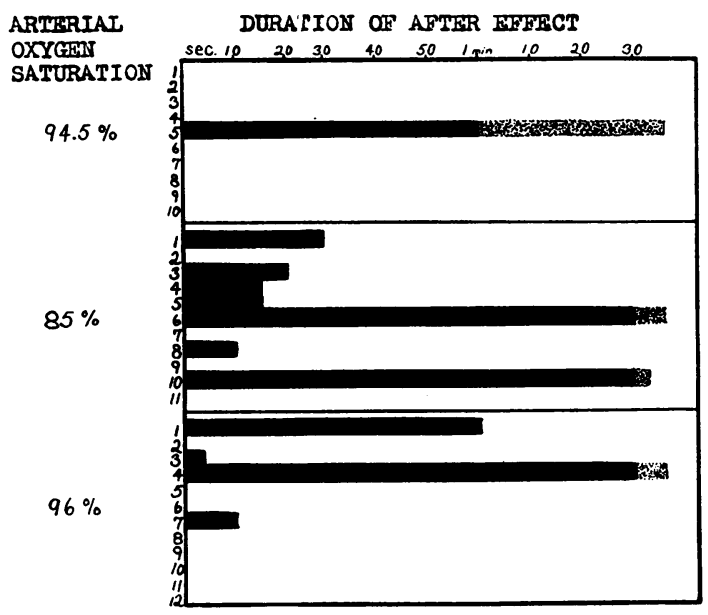

Fig. 1. Experiment 10. The Response of the Auricles (Vagal Tone Completely Removed) to Faradic Stimulation During Periods of Normal and Low Oxygen Saturation of the Blood

The chart is divided into three parts, the upper representing 10 trials with faradic stimulation, the arterial oxygen saturation being 94.5 . The middle part shows the result of 11 faradic stimulations, the blood oxygen saturation being 86 . The lower part shows the effect of 12 stimulations when the oxygen saturation of the blood was raised again to 96 . The black bars represent the duration of the after-effect, the stippled areas indicating that fibrillation lasted longer than the length of the black bar indicates and was ended by stimulating the auricles with a faradic current (except no. 10 of the middle block which ended spontaneously in two minutes). Note the increased tendency for the auricles to fibrillate during the anoxic period; and also the persistence of this tendency for a short time after the normal oxygen saturation of the blood was restored. 
This experiment demonstrates again the effect of anoxemia in predisposing the auricles to fibrillation. It shows also that following a period of anoxemia, the recovery process in the auricles is delayed, so that fibrillation may be produced with the blood fully saturated with oxygen, whereas previously under the same conditions of oxygen saturation no after-effects could be obtained. The experiment illustrates another finding that $I$ have noted, namely, that it is in early stages of anoxemia that the auricles exhibit a tendency to develop after-effects; generally the late effect of anoxemia is to inhibit the onset of fibrillation.

TABLE 5

Dog 35. Vagus nerves tied; no atropine used

\begin{tabular}{c|c|c|c}
\hline \multirow{2}{*}{$\begin{array}{c}\text { Oxygen saturation of } \\
\text { blood }\end{array}$} & $\begin{array}{c}\text { Number of faradic } \\
\text { stimulations }\end{array}$ & \multicolumn{2}{|c}{ Duration of after-effect } \\
\cline { 2 - 3 } per cent & & 15 to 59 seconds & 1 minute or longer \\
100 & $50(0)^{*}$ & 0 & \\
73 & $8(0)$ & 1 & 0 \\
100 & $5(3)$ & 0 & 0 \\
73 & $5(5)$ & 2 & 3 \\
100 & $4(4)$ & 0 & 2 \\
$65 \dagger$ & $7(7)$ & 0 & 0 \\
\hline
\end{tabular}

* The figures in the parentheses indicate the number of times vagal stimulation was used together with faradic stimulation.

† Approximately.

The findings in these and other experiments afford an explanation for the one instance (experiment 22), in which there was a greater tendency for fibrillation to appear when the blood was normally saturated with oxygen than when anoxemia was present. In this case, a moderate anoxemia ( 80 per cent saturation) had been present for slightly more than two hours. ${ }^{4}$ A high oxygen mixture was then given, and faradic stimulation produced three short and two long after-effects in thirty trials. On lowering the oxygen saturation of the blood to 80 per cent, fifty trials caused only one long after-effect. The reason for this exceptional result is indoubtedly due to the fact that functionally the auricles during the period when the blood was normally saturated, were in a state of mild anoxemia, due to incom-

${ }^{4}$ During this time other observations were being made. 


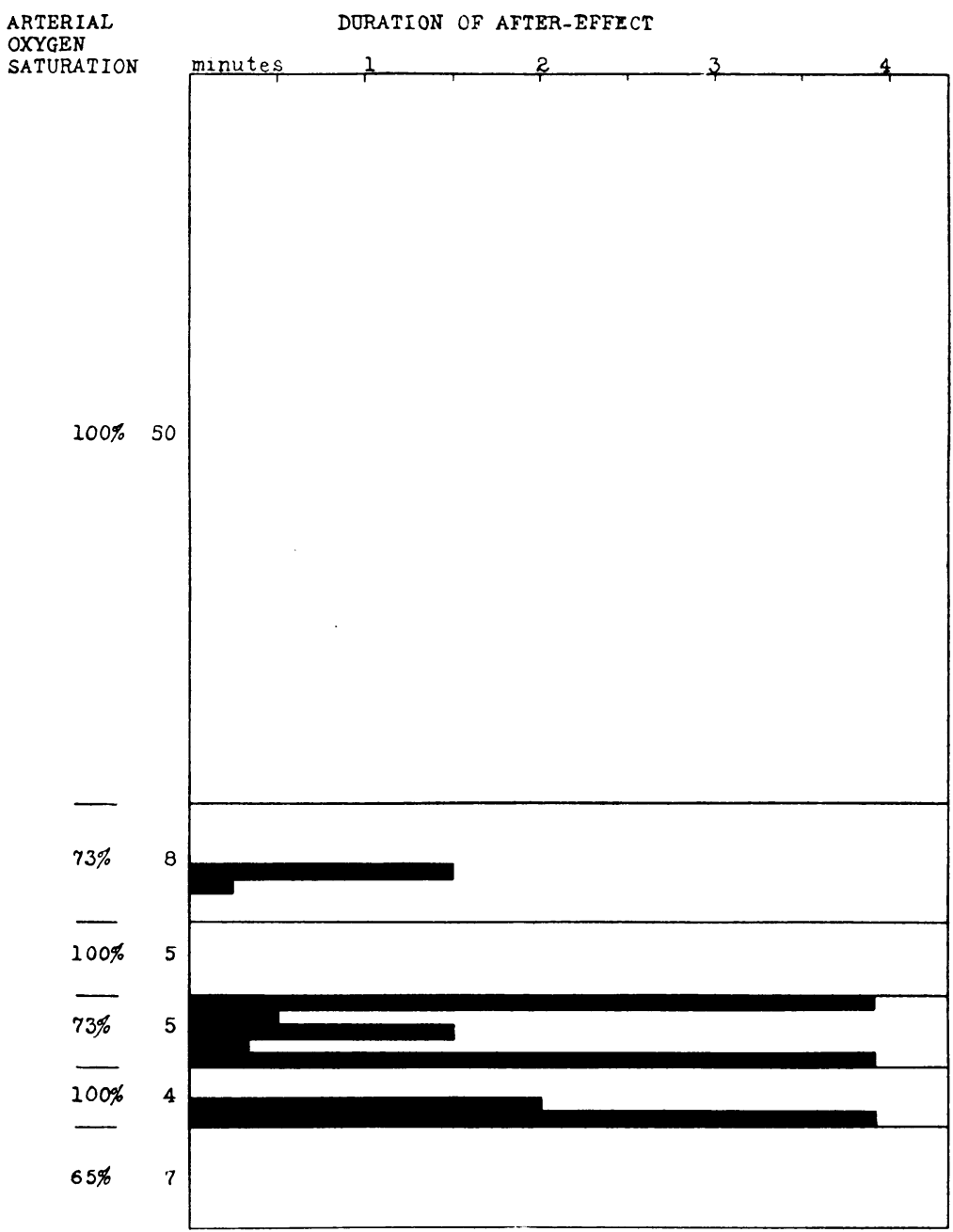

Fig. 2. Experiment 35. The Response of the Atricles to Faradic Stimllation, Vagal Tone Largely Eliminated, During Periods of Normal and Low Oxygen Saturation of the Blood

On the left hand side of the chart are given the percentages of oxygen saturation of the arterial blood and the number of faradic stimulations during the different periods. The width of a single black bar represents a single after-effect, its length showing the duration of the after-effect. The stippled areas mean that the after-effect in these instances lasted longer than the chart indicates. Note the increased tendency for fibrillation to develop during the early periods of anoxemia (second and fourth blocks of the chart); the persistence of this tendency when normal saturation is restored after a preceding period of anoxemia (fifth block); and the failure of fibrillation to appear in the late stages of anoxemia (lowest block). 
plete recovery from the previously existent anoxemia of long duration. When the oxygen saturation of the blood was then reduced, the late effect of anoxemia was seen, that is to say, auricular fibrillation was hindered.

There were three experiments in which no difference could be detected in the response to faradic stimulation, either with normal or low oxygen mixtures. In two of these, no after-effect could be produced. It must be recalled that atropine places a serious obstacle in the way of producing auricular fibrillation by any method. Consequently, it does not necessarily follow that anoxemia was without effect in these two cases. It may well have been that the anoxemia could not overcome the antagonistic influence of atropine.

One may summarize the foregoing data by stating that anoxemia predisposes the auricles to fibrillation, even when vagal tone is completely removed. Recovery of the auricles from a previous period of anoxemia is not immediate, and after-effects may be observed in incompletely recovered auricles when the blood is normally saturated with oxygen, whereas previously, under the same conditions of oxygen saturation of the blood, after-effects may have been absent. Anoxemia exerts that influence on the auricles which predisposes to fibrillation, relatively early; and the late effect of anoxemia is to inhibit the development of auricular fibrillation.

The refractory period of the auricle. In seven experiments, determinations of the refractory period of the auricles were made during periods of normal and diminished oxygen saturation of the blood. It was found that anoxemia first decreased the refractory period, but later increased it, bringing on various degrees of intra-auricular block. In table 6 are the data of an experiment illustrating the diminution of the refractory period by anoxemia.

In this table are given the figures for the latest ineffective ${ }^{5}$ and the earliest effective shocks, the calculated refractory period being

"In this and following tables, the "latest ineffective stimulus" does not actually represent the longest interval between rhythmic and interrupting shocks at which a response occurs. It represents the interval below which all other interrupting stimuli are ineffective. A certain amount of overlapping of effective and ineffective shocks is prone to occur when the auricles ase atropinized, particularly at the higher rates of driving. 


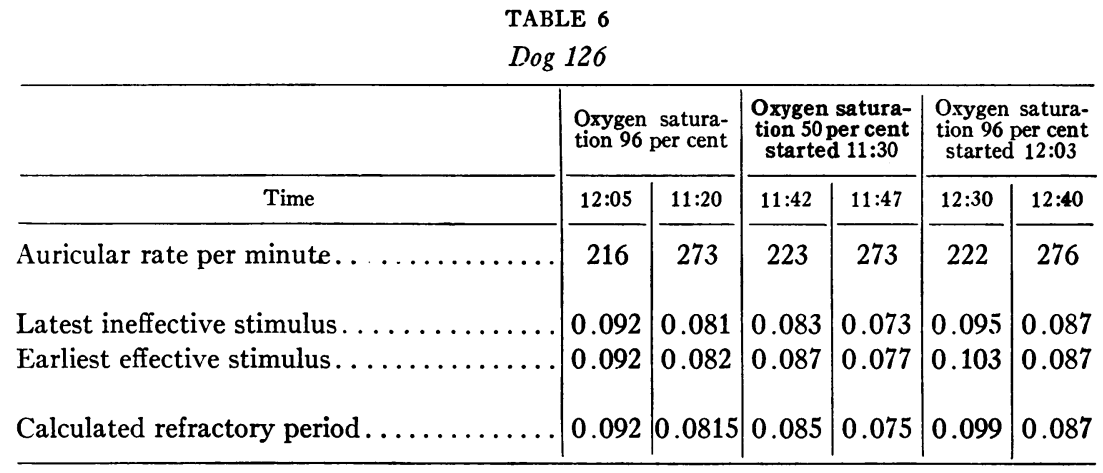

TABLE 7

Dog 30. Auricular rate 295 per minute

\begin{tabular}{l|c|c|c|c}
\hline Time & Oxygen saturation & $\begin{array}{c}\text { Latest ineffective } \\
\text { stimulus }\end{array}$ & $\begin{array}{c}\text { Earliest effective } \\
\text { stimulus }\end{array}$ & $\begin{array}{c}\text { Calculated } \\
\text { refractory period }\end{array}$ \\
\cline { 1 - 2 } & per cent & & & \\
& 100 & 0.090 & 0.097 & 0.0935 \\
$12: 14$ & 73 & 0.076 & 0.078 & 0.077 \\
$12: 38$ & 73 & 0.095 & 0.101 & 0.098 \\
$12: 41^{*}$ & $\dagger$ & 0.106 & $\ddagger$ & $>0.106$ \\
$12: 46$ & $\dagger$ & & & \\
\hline
\end{tabular}

* Two minutes after appearance of ventricular fibrillation.

$\dagger$ No figures are given for the percentage of arterial oxygen saturation as this must have decreased very rapidly during ventricular fibrillation.

† There were no instances in which an interrupting shock was preceded by two effective rhythmic shocks, a necessary precaution in the determination of the refractory period (Lewis, Drury and Bulger, 1921). Consequently, no determination was made for the earliest effective stimulus at this time.

TABLE 8

Dog 27. Auricular rate 221. Refractory period at rate of 214 was 0.1095 with normal arterial oxygen saturation

\begin{tabular}{c|c|c|c}
\hline & \multicolumn{3}{|c}{$\begin{array}{c}\text { Oxygen saturation } \\
\text { Started 1:35 per cent }\end{array}$} \\
\cline { 2 - 4 } Time & $\begin{array}{c}\text { Latest ineffective } \\
\text { stimulus }\end{array}$ & $\begin{array}{c}\text { Earliest effective } \\
\text { stimulus }\end{array}$ & $\begin{array}{c}\text { Calculated refractory } \\
\text { period }\end{array}$ \\
\hline $1: 57$ & 0.093 & 0.093 & 0.093 \\
$2: 04$ & 0.082 & 0.090 & 0.086 \\
$2: 10$ & 0.087 & 0.091 & 0.089 \\
$2: 19$ & 0.098 & 0.098 & 0.098 \\
$2: 32$ & 0.092 & 0.100 & 0.096 \\
$2: 40$ & Intra-auricular & - & - \\
\hline
\end{tabular}


taken as the average of the two figures. In this experiment, shortly after producing a severe anoxemia, the refractory period decreased slightly but definitely, returning to figures above those originally obtained, when the normal oxygen saturation of the blood was restored.

In tables 7 and 8 are recorded two further experiments in which anoxemia was continued until the animals died. In both there is seen a fall, then a rise in the refractory period during anoxemia. In each of these experiments the refractory period increased only when the animal was in very poor condition.

TABLE 9

\begin{tabular}{c|c|c}
\hline Time & Oxygen saturation of blood & Rate of auricle \\
\cline { 2 - 3 } $12: 23$ & per cent & per minute \\
$12: 25$ & 100 & 659 \\
$12: 36$ & 50 & \\
$12: 40$ & 50 & 621 \\
$12: 45$ & 50 & 608 \\
$12: 50$ & 50 & 612 \\
$12: 55$ & 50 & 614 \\
$1: 00$ & 50 & 590 \\
$1: 05$ & 50 & 549 \\
$1: 05.30$ & 50 & 480 \\
$1: 06$ & 50 & 465 \\
$1: 07$ & 50 & 440 \\
& 50 & Normal mechanism* \\
\hline
\end{tabular}

* The animal died a few minutes after the reversion to normal mechanism.

In two of the four remaining experiments, there was a decrease in the refractory period, which occurred a short time after a moderate anoxemia was started. In the other two experiments only the stage of increase in the refractory period was obtained; in one the anoxemia was severe ( 47 per cent arterial oxygen saturation); in the other, the anoxemia was mild (oxygen saturation, 89 per cent), but of relatively long duration, lasting almost two hours.

The data on the effect of anoxemia on the refractory period of the auricle are admittedly insufficient to give a complete picture since the rhythmic rates were relatively low in the determinations which I made. They are presented because they are consistent, in a general way, with the other observations on the effect of anoxemia. 
Effect of anoxemia on auricular fibrillation already present. On several occasions the effect of anoxemia on the rate of a circus movement was noted. Experiment 21 (table 9) is illustrative of the others.

Eleven minutes after the onset of anoxemia, there was a slight decrease in the rate of fibrillation, from 659 to 640 per minute; the level then remained about the same for fourteen minutes more. The rate then fell again, gradually at first, but very rapidly before the spontaneous reversion to the normal mechanism took place.

In one animal (experiment 35, table 10) flutter was produced shortly after anoxemia was induced. The rate of the auricle diminished

TABLE 10

Dog 35. Vagus nerves tied; atropine not used. Auricular flutter started at 12:05

\begin{tabular}{c|c|c}
\hline Time & Oxygen saturation of blood & Auricular rate \\
\cline { 2 - 3 } $12: 06$ & per cent & per minute \\
$12: 08$ & 73 & 600 \\
$12: 11$ & 73 & 590 \\
$12: 18$ & 73 & 582 \\
$12: 29$ & 73 & 580 \\
$12: 30$ & 73 & 582 \\
$12: 31$ & 100 & \\
$12: 33$ & 100 & 583 \\
$12: 40$ & 100 & 588 \\
$12: 46$ & 100 & 588 \\
$12: 50$ & 100 & 588 \\
$12: 50.15$ & 100 & 587 \\
& 100 & Normal mechanism \\
\hline
\end{tabular}

gradually from 600 to 582 per minute within five minutes and the rate remained constant for eighteen minutes. A high oxygen mixture was then given. The rate rose to 588 within three minutes, remained at this level for seventeen minutes and almost immediately after the last record was taken (the rate of which was 587) the normal mechanism was restored.

\section{DISCUSSION}

The results which were obtained with faradic stimulation during anoxemia indicate definitely that anoxemia predisposes the auricles to fibrillation, and this effect is the more striking in that it may overcome the powerfully antagonistic influence of atropine. I have 
purposefully stated that anoxemia predisposes the auricles to fibrillation: in none of these experiments has auricular fibrillation appeared spontaneously. Apparently some other factor besides anoxemia is necessary to initiate this arrythmia. In these observations, using the normal heart of the dog, this additional factor was the rapid stimulation of the auricles by a faradic current. What this second factor may be in the various clinical conditions in which anoxemia seems to play a rôle in causing transient auricular fibrillation, it is difficult to determine. Undoubtedly this influence differs in the individual case, although fundamentally its effect is probably always the same.

The development of a circus movement depends essentially upon shortening of the refractory period and lengthening of the rate of conduction, these occurring in such a fashion that the slowly travelling wave of excitation is always able to enter responsive muscular tissue, the refractory state having passed off. When the two factors of primary importance, namely refractory period and rate of conduction, are altered in such a way that they are mutually antagonistic, the outcome depends on the preponderating effect of one or the other of the actions. Thus, at high rates of stimulation atropine slows the rate of transmission of the excitation wave; but the opposite influence of the increased refractory period practically always predominates, and auricular fibrillation is hindered. Conversely, vagal stimulation increases the rate of transmission through the auricle at high rates of stimulation; but the absolute refractory period is shortened at the same time, and usually the appearance of auricular fibrillation is favored.

At first sight it would appear that the effect of anoxemia on the refractory period explains the type of response of the auricles to faradic stimulation in the early and late stages of anoxemia. It is in the early stage that the auricles show a predisposition to the development of fibrillation; and it is at this time that the refractory period is shortened. Likewise, the failure of fibrillation to appear (usually) in the late stages of anoxemia is apparently explained by the increase in the refractory period. Unfortunately, I have been unable to make determinations of the rate of transmission of the excitation wave through the auricular muscle, nor have I obtained satisfactory ob- 
servations on the effect of anoxemia on the refractory period at high rates of stimulation. I have pointed out elsewhere (Resnik, 1925a) that at relatively low rates of stimulation, auriculo-ventricular conduction may be quickened by anoxemia, whereas higher rates may cause conduction defects to appear. It is quite possible that the same events may take place in the refractory period of the auricles. Indeed, from the response to anoxemia of a circus movement already established (tables 9 and 10) it seems certain that the simple shortening of the refractory period cannot entirely account for the development of auricular fibrillation in the early stages of anoxemia; for if it were the case, the rate of the circulating rhythm should increase, for a short time at least, on changing from normal to low oxygen saturation of the blood. The shortening of the refractory period, if it were the only change to occur, should then permit the excitation wave to take a shorter path, this in turn allowing the excitation wave to traverse its course a greater number of times per minute. However, I have found that soon after diminishing the percentage of oxygen saturation of the blood, the rate of the circus movement decreased. Without a knowledge of the actual changes which occur in the refractory period and the rate of conduction when the auricles beat rapidly during anoxemia, it is useless to pursue the discussion. With the present data, one can state that, whatever may be the absolute alterations of refractory period and conduction, relatively the refractory period is decreased and conduction slowed, in the early stages of anoxemia, so that a circus movement is permitted to develop; in the late stages of anoxemia the reverse is true. ${ }^{6}$

\section{SUMMARY AND CONCLUSIONS}

1. The sino-auricular node is highly sensitive to anoxemia. There is at first a brief period during which the rate of impulse formation is

${ }^{6}$ No reference has been made as to the ultimate cause by which slowing of the rate of transmission of the excitation wave through the auricles may be brought about. Drury (1925) describing work by himself and Andrus has presented evidence to show that injury of the muscular tissue by various means causes the phenomenon known as "conduction with decrement" to appear. Whether this is true of conduction defects in the auricles due to anoxemia (and it is highly probable that they exist), I am unable to state. Since this paper was written, Drury's article has appeared in Heart, 1925, xii, 143. 
accelerated, followed rapidly by progressive slowing of the intrinsic rate of the heart.

2. Relatively low rates of rhythmic stimulation, anoxemia first reduces, then increases the absolute refractory period of the auricles.

3. Anoxemia diminishes the rate at which a circus movement travels throughout the auricle.

4. When vagal tone is removed, and the auricles are stimulated by a faradic current, the early effect of anoxemia is to predispose the auricles to fibrillation; the late effect is to inhibit the development of fibrillation. The mechanism by which anoxemia produces these effects is discussed.

\section{BIBLIOGRAPHY}

Boer, S. de.: Jour. Physiol., 1921, liv, 400. On the Fibrillation of the Heart. Cohn, A. E., and Jamieson, R. A.: Jour. Exp. Med., 1917, xxv, 65. The Action of Digitalis in Pneumonia.

Cole, R.: Nelson Loose-Leaf Living Medicine. New York, 1920, i, 232.

Dieuaide, F. R., and Turner, R. H.: Bull. Johns Hopkins Hosp., 1924, xxv, 411. Note on a Timing Device for the Determination of the Refractory Period of the Heart.

Drury, A. N.: Lecture, Johns Hopkins Medical Society, May 22, 1925, on "Conduction in Damaged Heart Muscle."

Lewis, T., Drury, A. N., and Bulger, H. A.: Heart, 1921, viii, 83. Observations upon Flutter and Fibrillation. Part VI. The Refractory Period and Rate of Propagation in the Auricle: Their Relation to Block in the Auricular Walls and to Flutter, etc.

Lewis, T., Drury, A. N., Wedd, A. M., and Iliescu, C. C.: Heart, 1921-22, ix, 207. Observations upon the Action of Certain Drugs upon Fibrillation of the Auricles.

Resnik, W. H.: Jour. Clin. Invest., 1924, i, 181. Transient Auricular Fibrillation Following Digitalis Therapy, with Observations upon the Response to Atropine.

Resnik, W. H.: (a), Jour. Clin. Invest. 1925, ii, 93. Observations on the Effect of Anoxemia on the Heart. I. Auriculo-ventricular Conduction.

Resnik, W. H.: (b) Jour. Clin. Invest. 1925, ii, 117. Observations on the Effect of Anoxemia on the Heart. II. Intraventricular Conduction.

Vaquez, H.: Les Arrythmies. Paris, 1911.

Winterberg, H.: Arch. f. des ges. Physiol., 1908, cxxxii, 361. Studien über Herzflimmern. II. Mitteilung. Über die Beeinflussung des Herzflimmerns durch einige Gifte. 